\title{
Modélisation de la dispersion des substances solubles ou pseudo-solubles dans un cours d'eau. Application au cas de la Seine à l'amont de Paris
}

\author{
A model of the dispersion \\ of soluble of pseudo-soluble substances in waterways. \\ Application to the case of the Seime upstream of Paris
}

\author{
Gérard Bujon \\ Compagnie Générale des Eaux*
}

\begin{abstract}
Avant-propos
par A. LESOUEF, Chef du Service Qualité des eaux et études, Agence financière de bassin Seine-Normandie
\end{abstract}

La mise au point du schéma d'aménagement des eaux de la Région Ile de France, actuellement en cours, fait l'objet d'un grand nombre d'études notamment en ce qui concerne la qualité des eaux de la Seine.

Chacun sait que la Seine (de même que la Marne et 1'Oise) est très largement utilisée pour l'alimentation en eau potable de l'agglomération parisienne. Les prises d'eau se répartissent d'amont en aval de Morsangsur-Seine jusqu'à Suresnes, c'est-à-dire jusqu'au coeur même de l'agglomération. On comprend donc que la maîtrise de la qualité des eaux du fleuve soit de première importance.

Mais il convient en outre que les eaux de la Seine soient d'une qualité compatible avec la survie piscicole et qu'elles ne soient pas source de nuisances pour l'environnement (aspect esthétique et absence d'odeurs désagréables).

Le respect des objectifs souhaitables de qualité n'est pas chose aisée compte tenu de la taille de l'agglomération et de la multiplicité des causes de dégradation: insuffisance de certains réseaux d'assainissement à l'origine de rejets urbains directs, déversements d'effluents industriels non épurés, déversements temporaires d'eau de ruissellement pluvial souvent fortement pollués et effluents de stations d'épuration dont la définition du niveau de traitement est un des buts des études.

La définition des mesures d'assainissement à adopter pour atteindre les objectifs précités implique, en premier lieu, la compréhension des relations de cause à effet entre rejets et qualité du milieu naturel. Il est vite apparu qu'il fallait pour cela disposer de modèles de

(*) détaché à l'Agence Financière de Bassin Seine-Normandie. qualité. Par souci d'efficacité il a été décidé d'opérer en deux étapes principales:

- recherche des relations rejets/qualité de l'eau hors période pluvieuse, les seuls rejets pris en compte étant ceux permanents de temps sec;

- étude des phénomènes se produisant par temps de pluie, tenant compte notamment des effluents provenant des déversoirs d'orage des réseaux unitaires

La première étape, actuellement terminée, a conduit à la mise au point d'un modèle de qualité de temps sec de Montereau au barrage de Poses qui a permis de définir les mesures d'assainissement à prévoir tant du point de vue réseaux que du point de vue stations d'épuration.

Pour la seconde étape qui fait intervenir des rejets non permanents et l'existence d'écoulements transitoires, il a paru nécessaire de tenir compte de phénomènes de dispersion turbulente dont l'importance avait été mise en évidence dans des expériences de traçage réalisées en Seine par la Compagnie Générale des Eaux lors d'études de sécurité d'alimentation en eau pilotées par l'Agence.

Le modèle de dispersion DISPERSO mis au point et décrit par $M$. BUJON dans l'article qui fait suite s'intègre dans ces études de seconde phase et il constitue en outre un outil de prévision très utile pour déterminer les conséquences des accidents éventuels de pollution dans un cours d'eau.

L'utilisation de modèles mathématiques de qualité d'eau suscite parfois du scepticisme chez certains techniciens sanitaires qui les connaissent encore mal. Ces modèles constituent pourtant le seul moyen rationnel de quantifier l'importance des phénomènes en rivière 
et de motiver raisonnablement des investissements qui, en matière de réseaux d'assainissement et de stations d'épuration, sont toujours très lourds.

Les techniciens ne devraient pas avoir pour préoccupation l'utilité d'un modèle de qualité mais plutôt le choix du "bon" modèle, facteur de progrès. Celui-ci doit en effet être suffisamment précis pour l'application considérée et ne pas atteindre une complexité monstrueuse. De ce point de vue DISPERSO donne des résultats tout à fait satisfaisants et "tourne" sur un miniordinateur de 16 Kilooctets de mémoire centrale...

\begin{tabular}{|c|c|c|c|c|c|}
\hline \multicolumn{6}{|c|}{ Liste des symboles } \\
\hline & & Dimension & $t$ & Temps............. & $\mathrm{T}$ \\
\hline$A$ & Durée de l'injection . . . . . . & $\mathrm{T}$ & $U$ & Vitesse ponctuelle moyenne & \\
\hline$B$ & Largeur du cours d'eau . . . . . & $\mathrm{L}$ & & de l'écoulement. . . . . . . . . & L.T -1 \\
\hline$C_{0}$ & $\begin{array}{l}\text { Concentration de la solution } \\
\text { du traceur } \ldots \ldots \ldots \ldots \ldots\end{array}$ & M.L -3 & $U_{0}$ & $\begin{array}{l}\text { Vitesse maximale d'écou- } \\
\text { lement sur l'axe longitudinal. }\end{array}$ & L.T -1 \\
\hline C & $\begin{array}{l}\text { Concentration du traceur ou } \\
\text { du polluant dans le cours d'eau. }\end{array}$ & $M . L-3$ & $x, y, z$ & $\begin{array}{l}\text { Distances dans le sens longi- } \\
\text { tudinal, vertical ou transversal. }\end{array}$ & $L$ \\
\hline$D T$ & Pas de temps. . . . . . . . . & $\mathrm{T} \ldots$ & $X_{p}$ & Position longitudinale du & \\
\hline$E_{x}, E_{y}, E_{z}$ & $\begin{array}{l}\text { Coefficient de dispersion tur- } \\
\text { bulente dans le sens longi- } \\
\text { tudinal, vertical et transversal. }\end{array}$ & $\mathrm{L} 2 . \mathrm{T}-1$ & $Z_{0}$ & $\begin{array}{l}\text { point étudié } \ldots \ldots \ldots \ldots \ldots \\
\text { Cote latérale du point d'injec- } \\
\text { tion }(O \text { au milieu }) \ldots \ldots \ldots \ldots\end{array}$ & $L$ \\
\hline$G$ & $\begin{array}{l}\text { Coefficient de décroissance li- } \\
\text { néaire de vitesse d'écoulement }\end{array}$ & $\mathrm{T}-1$ & $Z_{p}$ & $\begin{array}{l}\text { Cote latérale du point étudié } \\
(O \text { au milieu }) \ldots \ldots \ldots \ldots\end{array}$ & $L$ \\
\hline$H$ & Hauteur d'eau. . . . . . . . . & $\mathrm{L}$ & $Z R A(R)$ & Distance latérale source-image- & \\
\hline$K$ & $\begin{array}{l}\text { Coefficient de disparition } \mathrm{du} \\
\text { traceur (ou du polluant). . . }\end{array}$ & $\mathrm{T}-1$ & & $\begin{array}{l}\text { point étudié pour une ré- } \\
\text { flexion d'ordre } R \text { selon }\end{array}$ & \\
\hline$m$ & $\begin{array}{l}\text { Masse de traceur (ou de } \\
\text { polluant) } \ldots \ldots \ldots \ldots \ldots\end{array}$ & M & & $\begin{array}{l}\text { un cheminement de type } A \\
(O \text { au milieu }) \ldots \ldots \ldots \ldots\end{array}$ & $\mathrm{L}$ \\
\hline$N$ & Nombre de pas d'intégration. & - & $Z R B(R)$ & Distance latérale source- & \\
\hline$P$ & $\begin{array}{l}\text { Rapport du débit final au dé- } \\
\text { bit initial de l'injection. . . . }\end{array}$ & - & & $\begin{array}{l}\text { image-point étudié pour } \\
\text { une réflexion d'ordre } R \text { selon }\end{array}$ & \\
\hline$Q_{a}$ & Débit d'un affluent . . . . . . & L3.T -1 & & le cheminement de type $B$ & \\
\hline$Q_{0}$ & Débit initial de l'injection. & L3. T -1 & & $(O$ au milieu $) \ldots \ldots \ldots \ldots$ & $L$ \\
\hline$R$ & Numéro d'ordre de la ré- & & $\tau$ & Temps courant . . . . . . . & \\
\hline & flexion $\ldots \ldots \ldots \ldots$ & - & $\varphi$ & Flux de polluant ou de traceur & $\mathrm{M} \cdot \mathrm{T}-1$ \\
\hline
\end{tabular}

\section{Introduction}

Le transfert et la dispersion de substances solubles dans les cours d'eau sont des phénomènes susceptibles d'intéresser nombre de techniciens du domaine de l'eau :

- les Producteurs d'eau potable tout d'abord, dont la qualité de la matière première - souvent de l'eau de surface - dépend des injections permanentes ou temporaires effectuées en amont de leur prise d'eau,

- mais aussi les spécialistes de l'épuration et de la protection de l'environnement qui doivent se préoccuper du devenir et de l'impact du déversement d'effluents plus ou moins épurés dans les rivières.

Quand les rejets existent déjà, on peut aisément apprécier leurs conséquences grâce à des mesures dans le cours d'eau mais lorsqu'il s'agit de déversements projetés ou encore d'éventualités de déversements accidentels, on ne peut prévoir ce qui peut se passer sans disposer de méthodes de calcul, on dit aussi de modèles mathématiques de simulation ou de prévision.

Il convient d'ajouter cependant que la mise au point de ces modèles ne peut se faire sans expériences préalables d'injection d'un traceur de pollution bien choisi, suivies de mesures dans le cours d'eau de façon à pouvoir déterminer les coefficients spécifiques qui interviennent dans les calculs.
Une étude bibliographique montre que les travaux publiés sur le sujet sont relativement récents, vingt à trente ans au plus, les premiers étant ceux de G.I. Taylor qui a ouvert la voie ensuite à plusieurs autres chercheurs. L'intérêt des recherches a été certainement accru par l'apparition de problèmes de pollution de rivières de plus en plus fréquents. Cependant les résultats obtenus n'ont pas réellement franchi les frontières du cercle restreint de spécialistes de l'hydraulique qui se sont intéressés à la question et ce d'autant que l'utilisation pratique des modèles proposés nécessite (comme on le verra plus loin) des moyens de calcul très performants qui n'ont été disponibles que plus tard.

Les expériences de traçage en Seine effectuées par la Compagnie Générale des Eaux à l'amont de Paris, dans le cadre d'études de sécurité de l'alimentation en eau et pilotées par l'Agence de Bassin Seine Normandie, constituent une excellente occasion de mettre à l'épreuve les modèles en cause et de chercher à mettre au point un outil de prévision qui pourrait être très utile en cas de pollution accidentielle du fleuve.

\section{Etablissement du modèle de dispersion}

\section{Brève analyse physique du phénomène}

Lorsqu'un milieu quelconque présente des hétéro- 
généités de concentration, il peut s'établir des transferts de masse plus ou moins rapides des zones les plus concontrées vers celles de concentration les plus faibles. Ce phénomène de transfert appelé diffusion moléculaire est gouverné par les lois de Fick.

La diffusion moléculaire intervient naturellement dans des milieux liquides, mais lorsque ces derniers sont en mouvement, d'autres phénomènes plus intenses rendent ses effets pratiquement négligeables. Dans ce cas en effet il existe en chaque point du liquide en mouvement des fluctuations aléatoires de vitesse d'écoulement qui provoquent une convection différentielle et par conséquent une dispersion des éléments dont l'eau est éventuellement chargée. Le phénomène existe déjà lorsque l'écoulement est laminaire mais en régime turbulent il s'y ajoute, du fait même de la turbulence, un effet de dispersion complémentaire appelé dispersion turbulente.

L'expérience montre que l'on peut décrire les phénomènes de dispersion par convection différen. tielle et de dispersion turbulente à l'aide de lois de même type que celle de Fick mais en utilisant, au lieu des coefficients de diffusion moléculaire, des coefficients de dispersion d'un ordre de grandeur très supérieur.

Ainsi la quantité de matière $d m$, transitant dans le temps $d t$, entre deux surfaces $S$ équidistantes, espacées de $d \ell$, entre lesquelles existe une différence de concentration $d C$, s'écrit :

$$
d m=E . S . \frac{d C}{d \ell} \cdot d t
$$

$E$ étant un coefficient de dispersion dans la direction normale aux deux surfaces. (Dimension $L^{2} / T$ ).

\section{Equation générale de la dispersion}

Soit un liquide s'écoulant en régime turbulent et transportant un composé soluble ou pseudo soluble. Les trois composantes du coefficient de dispersion dans le sens du mouvement, dans le sens transversal et dans le sens vertical sont supposés être des constantes.
Par ailleurs le champ des vitesses d'écoulement est supposé décroître linéairement tant dans le sens transversal que vertical à partir d'un point de vitesse maximale situé en surface et sur l'axe longitudinal de l'écoulement.

Cette hypothèse se trouve étayée par les résultats de mesure de vitesse disponibles qui montrent que dans le cas de la Seine, la variation transversale et verticale des vitesses peut approximativement être décrite par une loi du premier degré, au moins si l'on néglige les variations rapides au voisinage immédiat des berges et du fond.

Ainsi l'origine des distances transversales et verticales étant l'axe longitudinal de l'écoulement en surface, la vitesse $U$ en un point de coordonnées $(y, z)$ sera donnée par:

$$
U=U_{0}+G_{y} y+G_{z} \cdot|z|
$$

- $U_{0}$ étant la vitesse sur l'axe,

- $G_{y}$ et $G_{z}$ deux coefficients de décroissance linéaire de dimension $T^{-1} \cdot\left(G_{y} \leqslant 0\right.$ et $\left.G_{z} \leqslant 0\right)$

La figure 1 illustre l'hypothèse retenue pour le profil transversal et vertical des vitesses.

La loi de Fick, généralisée à des écoulements turbulents étant admise, un simple bilan massique dans un parallélépipède élémentaire dont l'une des faces se trouve dans le plan d'une section droite à l'écoulement permet d'établir l'équation générale de la dispersion.

Il suffit pour cela d'écrire que dans le temps $d t$, la différence des masses entrantes et sortantes correspond à la variation de masse dans le parallélépipède. $E_{x}, E_{z}$ et $E_{y}$ étant les composantes précitées de la dispersion et $C$ la concentration du produit, on obtient ainsi :

$$
\begin{aligned}
E_{x} \frac{\partial^{2} C}{\partial x^{2}} & +E_{z} \frac{\partial^{2} C}{\partial z^{2}}+E_{y} \frac{\partial^{2} C}{\partial y^{2}} \\
& -\left(U_{0}+G_{z} \cdot|z|+G_{y} y\right) \frac{\partial C}{\partial x}-\frac{\partial C}{\partial t}=0
\end{aligned}
$$

La relation (3) ci-dessus est établie en supposant que le composé dont on étudie la dispersion est conser-

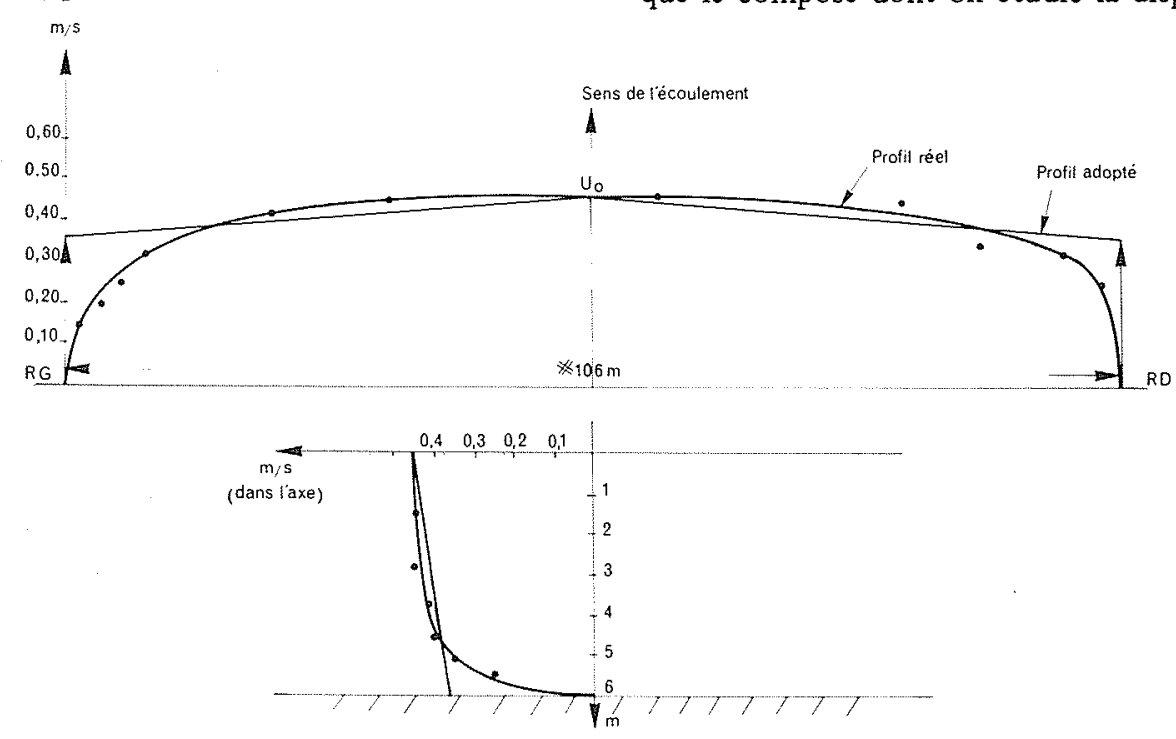

Figure 1. - Profils transversal et vertical des vitesses à Paris - Pont de Solférino - le 24 août 1979. 
vatif. Dans le cas contraire, il faut tenir compte de la perte correspondante dans le calcul de la variation de masse au sein du parallélépipède élémentaire.

On peut admettre que la concentration de la substance étudiée évolue dans le temps selon une réaction d'ordre I, comme on le constate généralement lorsqu'il se produit une biodégradation. Alors l'expression (3) se transforme en:

$$
\begin{aligned}
E_{x} & \frac{\partial^{2} C}{\partial x^{2}}+E_{z} \frac{\partial^{2} C}{\partial z^{2}}+E_{y} \frac{\partial^{2} C}{\partial y^{2}} \\
& -\left(U_{0}+G_{z} \cdot|z|+G_{y} y\right) \frac{\partial C}{\partial x}-\frac{\partial C}{\partial t}-K C=0
\end{aligned}
$$

$K$ étant un coefficient de "disparition" de dimension $T^{-1}$

A ce point du raisonnement, on peut s'interroger sur l'intérêt de conserver un modèle tridimensionnel dans l'étude d'un cours d'eau comme la Seine, d'une profondeur de quelques mètres seulement, où l'homogénéisation dans le sens vertical est certainement très rapide. En outre les essais de traçage n'ont pas comporté de prélèvements à différentes profondeurs et il ne serait pas possible de caler un modèle à trois dimensions.

On supposera donc que la substance étudiée est injectée de manière homogène sur une verticale, ce qui conduit à supprimer les termes en $Y$ dans l'équation (4). Il reste :

$$
\begin{aligned}
E_{x} \frac{\partial^{2} C}{\partial x^{2}}+ & E_{z} \frac{\partial^{2} C}{\partial z^{2}} \\
& -\left(U_{0}+G_{z} \cdot|z|\right) \frac{\partial C}{\partial x}-\frac{\partial C}{\partial t}-K C=0
\end{aligned}
$$

La solution de cette équation différentielle se présente sous la forme de la convolution suivante:

$$
C(x, z, t)=\varphi\left(x_{0}, z_{0}, t\right) * R(x, z, t)
$$

$\varphi\left(x_{0}, z_{0}, t\right)$ étant la variation de flux du composé injecté en fonction du temps au point $\left(x_{0}, z_{0}\right)$ et $R(x, z, t)$ une fonction de transfert qui fait correspondre à cette variation "à l'origine" la variation de concentration $C(x, z, t)$ au point étudié du cours d'eau. Cette fonction est aussi appelée réponse impulsionnelle.

\section{Solution dans le cas d'une injection instantanée}

Un cas particulier est celui où l'injection d'une masse $m$ est instantanée et peut être assimilée à une impulsion de Dirac ; on peut alors écrire:

$$
C(x, z, t)=m \cdot R(x, z, t)
$$

On démontre par ailleurs que lorsque l'injection est effectuée de manière homogène sur une verticale de hauteur $H$ : on obtient : avec

$$
F^{2}=\frac{G_{z}^{2}}{12} \cdot \frac{E_{z}}{E_{x}}
$$

\section{Solution dans le cas d'une injection prolongée. Principe de superposition}

Si l'injection n'est pas instantanée le calcul de $C(x, z, t)$ peut être effectué en exprimant la convolution sous sa forme intégrale:

$$
\begin{aligned}
C_{(x, z, t)} & =\varphi\left(x_{0}, z_{0}, t\right) * R(x, z, t)= \\
& =\int_{0}^{T} \varphi\left(x_{0}, z_{0}, \tau\right) \cdot R\left[x, z_{,}(t-\tau)\right] \cdot d \tau
\end{aligned}
$$

avec $T=t$ si $t \leqslant A$

et $T=A$ si $t>A$

- $t$ étant le temps pour lequel la concentration au point $(x, z)$ est cherchée,

- $A$ la durée de la période d'injection et

- $\tau$ le temps courant compté à partir du début de l'injection.

Il n'est peut-être pas inutile de préciser la signification physique de l'intégrale (10); celle-ci traduit ce que certains appellent le principe de superposition.

L'injection au point $\left(x_{0}, z_{0}\right)$ est considérée comme une succession dans le temps d'impulsions de Dirac. A chacune de ces impulsions correspond une réponse impulsionnelle donnant au point $(x, z)$ et au temps $t$ une certaine valeur de concentration.

Les concentrations sont, comme le montre l'expression (7), proportionnelles à l'intensité des impulsions qui les ont générées et les différentes courbes concentrationtemps correspondant à chaque impulsion sont décalées l'une l'autre du temps qui sépare deux impulsions successives. Le principe de superposition suppose que la concentration au point considéré soit la somme de toutes les composantes impulsionnelles en ce point pendant la durée de l'injection.

La figure 2 montre la signification graphique de l'intégrale de convolution, la concentration cherchée correspondant à l'aire hachurée.

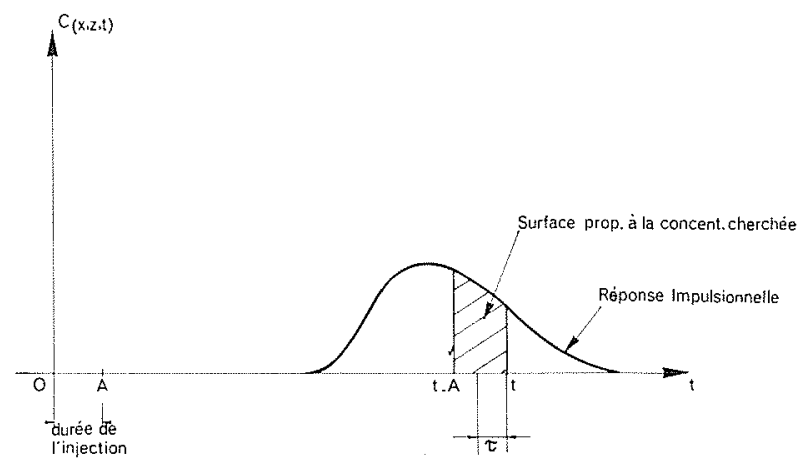

Figure 2. - Injection prolongée. Signification graphique de l'intégrale de convolution. cas $t>\mathrm{A}$

$$
R_{(x, z, t)}=\frac{1}{4, \pi, H\left(E_{x} \cdot E_{z}\right)^{1 / 2} t\left(1+F^{2} t^{2}\right)^{1 / 2}} \exp \left[-\frac{\left[x-\left(U_{0}+\frac{1}{2} G_{z}|z|\right) t^{2}\right]}{4 \cdot E_{x} \cdot t\left(1+F^{2} t^{2}\right)}-\frac{z^{2}}{4 \cdot E_{z} \cdot t}-K t\right]
$$




\section{Cas des écoulements de largeur finie - Méthode des sources-images}

La solution de l'équation différentielle bidimensionnelle qui a été examinée jusqu'à présent n'est utilisable que dans la partie de l'écoulement, à l'aval de l'injection, où le composé n'a pas encore atteint les berges. Pour s'affranchir de cette contrainte, on peut faire appel à la méthode des sources-images.

La condition d'absence de transport du composé en cause, hors du cours d'eau, peut être simulée en plaçant à terre, symétriquement à la source réelle par rapport à la berge, une source virtuelle identique à la source réelle. De cette manière, les concentrations de part et d'autre de la berge sont identiques et cela interdit tout transport dispersif du composé hors du milieu liquide.

On peut aussi considérer que cet artifice correspond à une réflexion du composé contre la berge: la part du composé qui, sans réflexion, sortirait de la phase liquide, est renvoyée dans l'écoulement et son effet s'ajoute en un point donné au composé qui y parvient directement.

Il faut bien entendu prévoir autant de sourcesimages sur une berge que sur l'autre et en principe, le nombre de ces sources-images de chaque côté est infini.

Sayre et Chang ont proposé deux relations pour déterminer la distance latérale sources-images/point de l'écoulement considéré.

$\mathrm{Si}$ l'origine des coordonnées est l'axe longitudinal de l'écoulement, $Z_{0}$ la position de la source réelle, $Z_{p}$ celle du point étudié, $B$ la largeur de l'écoulement et $R$ le numéro d'ordre de la réflexion considérée, cette distance est donnée par:

$$
Z R A(R)=R \cdot B-Z_{0}+Z_{p},(-1)^{R}
$$

pour un cheminement du composé avec première réflexion en rive droite et

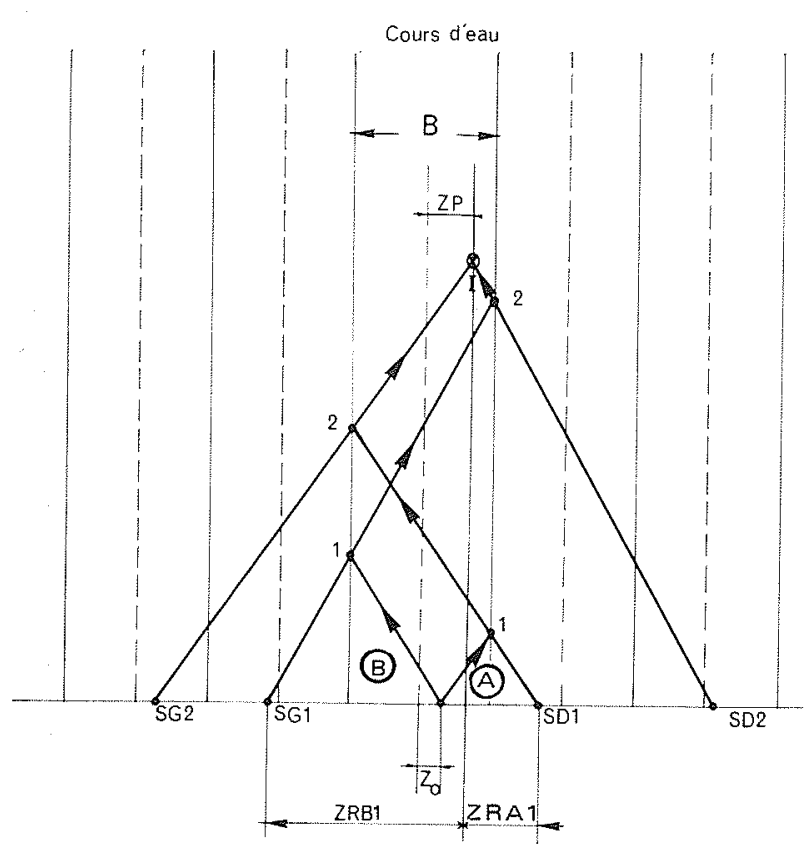

Figure 3. - Dispersion dans un cours d'cau de largeur finic. Sources: images dans le cas de deux réflexions.

$$
Z R B(R)=R \cdot B+Z_{0}-Z_{p}(-1)^{R}
$$

pour un cheminement du composé avec première réflexion en rive gauche.

La figure 3 illustre dans le cas de deux réflexions seulement, la position des sources-images.

En définitive, dans le cas d'une injection prolongée, effectuée dans un écoulement de largeur finie, en un point de coordonnées $\left(O, Z_{0}\right)$; la concentration au temps $t$ au point aval $\left(X_{p}, Z_{p}\right)$ résulte de l'effet combiné des impulsions de la source réelle et des sources-images, d'où :

$$
\begin{aligned}
& C_{T}\left(X_{p}, Z_{p}, t\right)=C\left(X_{p}, Z_{p}-Z_{0}, t\right) \\
& \quad+\sum_{R=1}^{R=\infty}\left[C\left(X_{p}, Z R A(R), t\right)+C\left(X_{p}, Z R B(R), t\right)\right]
\end{aligned}
$$

La fonction $C$ étant l'expression intégrale (10) dans laquelle il convient de donner à $z$ les valeurs appropriées, soit $z_{p}-z_{0}$, soit $Z R A(R)$, soit $Z R B(R)$.

L'expression (13) serait inutilisable s'il était nécessaire de tenir compte d'un nombre infini de réflexions. En pratique la somme peut être limitée aux quatre ou cinq premiers termes de $R$. On verra dans la suite que les calculs effectués au cours de la présente étude, confirment cette affirmation.

\section{Elaboration d'un programme de calcul}

Le modèle décrit ci-dessus a donné lieu à l'élaboration d'un programme de calcul mettant en œuvre les relations $8,9,10,11,12,13$, qui a été baptisé "DISPERSO".

L'intégrale (10) n'ayant pas de solution analytique, le calcul de $C(x, z, t)$ requiert une intégration numérique. Or est ainsi amené à discrétiser l'expression $(8) ; D T$ étant le pas d'intégration choisi, $T$ la borne supérieure de l'intégrale, le nombre de pas d'intégration $N$ est pris égal à l'entier le plus proche du rapport $\frac{T}{D T}$.

Pour l'intégration, on a retenu la méthode du trapèze. L'opération consiste donc très simplement à calculer pour la source réelle et pour les sources-images, une série de surfaces élémentaires trapézoïdales puis à en faire la somme.

En ce qui concerne le flux de la substance injectée dans le cours d'eau, on a envisagé trois éventualités:

- Cas des traçages: le débit et la concentration restent constants au cours de l'injection ;

- Cas des accidents de pollution: la concentration reste constante mais le débit décroit au cours de l'injection. On a étudié l'hypothèse d'une décroissance linéaire correspondant au cas fréquent de la vidange d'une cuve à section horizontale constante;

- Cas des affluences: le débit peut être considéré comme constant au cours du passage de la vague de pollution mais la concentration varie continuellement dans le temps.

Les deux premiers cas peuvent être traités par une relation unique en introduisant comme variable le rapport 
$P=$ Débit final/Débit initial de la solution injectée.

Le débit initial de la solution injectée étant $Q_{0}$ et $C_{0}$ sa concentration, l'intégrale discrétisée à faire intervenir dans le programme de calcul s'écrit :

$$
\begin{aligned}
& C(x, z, t)=\frac{C_{0}, Q_{0}}{4 . \pi \cdot H\left(E_{x} \cdot E_{z}\right)^{1 / 2}} \sum_{N=0}^{N} \\
& \frac{1}{2}[f(t-N . D T)+f\{t-(N+1) . D T\}] D T
\end{aligned}
$$

avec

$$
\begin{gathered}
f(t-N . D T)=\frac{1+\frac{P-1}{A}(t-N . D T)}{(t-N . D T)\left[1+F^{2}(t-N . D T)^{2}\right]^{1 / 2}} \\
\exp \left[-\frac{\left[x-\left(U_{0}+\frac{1}{2} G_{z}|z|\right)(t-N . D T)\right]^{2}}{4 E_{x}(t-N . D T)\left[1+F^{2}(t-N . D T)^{2}\right]}\right. \\
\left.-\frac{z^{2}}{4 E_{z}(t-N . D T)}-K(t-N . D T)\right]
\end{gathered}
$$

et $f(t-(N M) . D T)=d^{\circ},(t-N . D T)$ étant remplacé par $(t-(N+1) . D T)$

Pour le troisième cas, l'expression suivante est à utiliser, $Q A$ étant le débit constant de la solution et $\operatorname{Co}(N . D T)$ sa concentration:

$$
\begin{aligned}
C(x, z, t) & =\frac{Q_{A}}{4 . \pi \cdot H\left(E_{x} E_{z}\right)^{1 / 2}} \sum_{N=0}^{N} \\
& \frac{1}{2}[f(t-N . D T)+f\{t-(N+1) . D T\}] . D T
\end{aligned}
$$

avec

$$
\begin{aligned}
f(t-N . D T) & =\frac{C_{0}(N . D T)}{(t-N . D T)\left[1+F^{2}(t-N . D T)^{2}\right]^{1 / 2}} \\
\exp & {\left[-\frac{\left[x-\left(U_{0}+\frac{1}{2} G_{z}|z|\right)(t-N . D T)\right]^{2}}{4 E_{x}(t-N . D T)\left[1+F^{2}(t-N . D T)^{2}\right]}\right.} \\
& \left.-\frac{z^{2}}{4 E_{z}(t-N . D T)}-K(t-N . D T)\right]
\end{aligned}
$$

et $f(t-(N+1) . D T)=d^{\circ},(t-N . D T)$ étant remplacé par $(t-(N+1) . D T)$

II n'est naturellement pas envisageable d'effectuer à la main (ou même avec une simple calculatrice électronique) le nombre important d'opérations que nécessite le calcul de $C(x, z, t)$ et le recours à l'ordinateur s'impose. Un programme de calcul en langage BASIC a donc été établi à cette fin.

Ce programme de calcul a été conçu de manière à ce que les données de concentration $C_{0}(N . D T)$ puissent être introduites soit par l'intermédiaire d'un clavier, soit par la lecture d'une bande magnétique.
Mais il a paru pratique, pour des simulations d'accident sur un affluent d'ordre quelconque, de prévoir également la possibilité d'un calcul enchaîné prenant automatiquement en compte comme données de concentration aux confluences le "pollutogramme" venant d'être calculé à l'extrémité du confluent correspondant.

La procédure retenue pour cela consiste à déterminer à cette extrémité, pour chaque temps de calcul, la concentration en plusieurs points répartis sur une transversale à l'écoulement puis à en effectuer la moyenne.

Il faut en outre déterminer le temps d'arrivée de la vague de pollution à la confluence, ce temps étant en effet celui du début de l'injection dans le cours d'eau récepteur. On y parvient en divisant le temps compris entre deux valeurs successives de calcul en un certain nombre de temps intermédiaires, en calculant par interpolation les concentrations correspondant à ces temps intermédiaires et en retenant, pour le début de la vague, le premier temps pour lequel la concentration devient supérieure à un seuil déterminé. Dans le programme de calcul, ce seuil a été choisi égal à $1 \mu \mathrm{g} / \mathrm{l}$.

Le programme fournit des résultats de calcul se présentant sous la forme de tableaux précédés d'un titre indiquant s'il s'agit de concentrations moyennes à l'aval d'un affluent ou du "pollutogramme" en un point donné de simulation.

Ces tableaux fournissent les dates et heures pour lesquelles les concentrations ont été calculées, l'origine des dates (jour 0) étant fixée au jour où l'injection parvient au milieu récepteur et les heures et minutes étant données en temps réel. Ils fournissent également le temps total écoulé depuis le début de cette injection et le temps partiel depuis l'arrivée de la vague de pollution dans le bief en cours d'étude.

Calage du modèle dans le cas de la Seine. (A l'amont de Paris)

\section{Objectif particulier du calage}

Compte tenu des particularités extrêmement changeantes d'un grand cours d'eau comme la Seine de l'amont à l'aval, il parait à priori très improbable que l'on puisse effectuer un calage satisfaisant du modèle sur une très longue partie de son cours. Tout au moins peut on espérer parvenir à un résultat convenable sur des tronçons de quelques dizaines de kilomètres, ce qui serait déjà très intéressant puisqu'en cas de pollution les prévisions les plus utiles portent sur des distances de cet ordre de grandeur, les pointes de teneur n'y étant pas encore très amorties.

Il faut donc envisager plusieurs calages par tronçons "homogènes" et ceci naturellement pour divers régimes hydrauliques. C'est là un travail de longue haleine qui déborde très largement l'objet de la présente communication et pour lequel d'ailleurs toutes les données nécessaires ne sont pas encore réunies.

L'objectif visé ici sera plus limité : il s'agira, une fois choisie une expérience de traçage parmi celles réalisées, de montrer qu'il est possible d'effectuer un calage 
acceptable du modèle à l'aide des résultats obtenus en certains points de mesure de cette expérience, c'est-àdire de déterminer la valeur des divers coefficients intervenant dans le modèle, de telle sorte que celui-ci permette de retrouver sans trop de divergences les résultats expérimentaux obtenus en d'autres points de ce traçage.

\section{Caractéristiques du traçage retenu}

On a retenu le traçage du 20 juillet 1981, l'injection étant réalisée dans la rivière Essonne à $200 \mathrm{~m}$ seulement de la confluence avec la Seine.

Au cours de l'expérience $19.8 \mathrm{~kg}$ de Rhodamine B ont été injectés en une heure sous forme d'une solution à $100 \mathrm{~g} / 1$ et la nappe de traceur a pu être suivie en Seine jusqu'à Ivry à $27,9 \mathrm{~km}$ à l'aval, des prélèvements intermédiaires ayant été effectués à Soisy-sur-Seine, $(3,2 \mathrm{~km})$, Ris Orangis $(7,1 \mathrm{~km})$, Viry-Chatillon $(9,65 \mathrm{~km})$ et Vigneux $(17,8 \mathrm{~km})$. Les prélèvements à Soisy-sur-Seine et à $\mathrm{Ris}$ Orangis ont été réalisés en trois points répartis sur une transversale à l'écoulement.

Ce traçage du 20 juillet 1981 présentait plusieurs caractéristiques favorables:

- Le débit de la Seine était relativement faible, $145 \mathrm{~m}^{3} / \mathrm{s}$, ce qui permettait de connaitre les coefficients de diffusion turbulente dans un domaine de débit intéressant quant à l'impact des pollutions accidentelles ; - L'injection dans un affluent à proximité de la confluence revenait à simuler d'une manière très satisfaisante l'hypothèse d'une injection homogène sur une verticale de la Seine. La seule particularité défavorable aurait pu être un certain étalement du traceur à son arrivée en Seine mais les mesures en ce point ont montré que le gros de la nappe y est passé en une heure environ et que le flux de Rhodamine $B$ est resté sensiblement constant pendant ce temps là.

- L'injection dans un affluent permettait de tester la possibilité qu'offre le programme de calcul d'effectuer des calculs enchaînés (le test devant toutefois être limité à des ordres de grandeur en raison du nombre insuffisant de mesures effectuées dans l'affluent).

\section{Méthodologie du calage}

Pour caler le modèle, on a considéré que l'injection de Rhodamine $B$ avait été effectuée directement en Seine.

Dans un premier temps, on a fait l'hypothèse que le traceur était conservatif et l'on a cherché à caler le modèle à Soisy-sur-Seine (Prélèvements "Rive Gauche" et "Milieu"), c'est-à-dire à une distance suffisamment faible de la source pour que la disparition éventuelle de la Rhodamine $B$ par quelque phénomène que ce soit (biodégradation notamment) soit négligeable. On a donné à $G$ une valeur comprise entre $-0,001 \mathrm{~s}^{-1}$ et $-0,0015 \mathrm{~s}^{-1}$ (fourchette que l'on peut déduire des valeurs de vitesse disponibles) et à partir de valeurs de coefficients de diffusion turbulente plausibles, on a fait varier alternativement $E_{x}$ et $E_{z}$ de manière à ce que les valeurs calculées se rapprochent des résultats expérimentaux.
On a ainsi pu déterminer les valeurs des coefficients de diffusion turbulente longitudinale et transversale, soit $8 \mathrm{~m}^{2} / \mathrm{s}$ et $0,09 \mathrm{~m}^{2} / \mathrm{s}$ respectivement, ou plus précisément les deux valeurs qui données aux coefficients $E_{x}$ et $E_{z}$ du modèle conduisent, dans le cas du traçage considéré, aux résultats de calcul les plus proches de la réalité.

On a cherché ensuite à déterminer la valeur du coefficient de disparition $K$ permettant de retrouver les teneurs maximales mesurées au point de prélèvement le plus éloigné soit à Ivry. Mais ce point a dû finalement être écarté car la masse de traceur qui y serait passée, selon les résultats du dosage, serait légèrement supérieure à la masse injectée à Corbeil. Cette anomalie qui résulte vraisemblablement de la difficulté de mesurer avec précision des teneurs aussi basses que celles rencontrées à Ivry (quelques $\mu \mathrm{g} / \ell$ au plus) a eu pour conséquence de déterminer le coefficient de disparition à l'aide des mesures faites à Vigneux.

Une valeur de $K$ ayant été ainsi trouvée, on a cherché par tatonnement, en jouant légèrement sur cette valeur et sur celle de $G$ et en vérifiant qu'il n'y avait pas d'incidence inacceptable pour les concentrations calculées au niveau de Soisy, à simuler d'une manière aussi proche que possible le profil de concentration mesuré à Vigneux.

On a trouvé en définitive $K=3 \cdot 10^{-6} \mathrm{~s}^{-1}$ et $G=8 \cdot 10^{-4} \mathrm{~s}^{-1}$.

\section{Présentation de quelques résultats de simulation et discussion}

Une illustration des simulations qui peuvent être faites après calage du modèle est donnée dans les figures 4 à 7 qui représentent les profils de concentration calculés aux points de prélèvements de Soisy (rive gauche et milieu), de Ris Orangis (milieu) et de Vigneux (rive droite). Dans chaque cas, les valeurs de concentration mesurées ont été pointées à titre de comparaison sur les graphiques de profils "calculés".

L'examen critique de ces figures doit être conduit en gardant constamment présent à l'esprit que les concentrations maximales du traceur dans l'eau de la Seine sont de l'ordre de quelques dizaines de $\mu \mathrm{g} / \ell$ aux points les plus proches de la source et de quelques $\mu \mathrm{g} / \ell$ seulement pour ceux les plus éloignés. Si performante que soit la technique de dosage de la Rhodamine $B$ et quels que soient les soins apportés à sa mise en cuvre, il parait raisonnable de ne voir dans certains résultats de mesure autre chose qu'un ordre de grandeur.

Ceci étant, on peut constater que le modèle permet de retrouver de manière satisfaisante l'allure des variations et l'ordre de grandeur des concentrations mesurées.

Les premières versions du modèle qui ne tenaient pas compte de l'existence du phénomène de disparition du traceur $(K=0)$ et dans lesquelles la vitesse d'écoulement était supposée uniforme $(G=0)$ ne permettaient d'effectuer des simulations satisfaisantes qu'en des points peu éloignés du point d'injection (une dizaine de $\mathrm{km}$ au plus dans les conditions du traçage du 20/7/81). Au-delà, les profils calculés se caractérisaient par des concentrations maximales exces- 

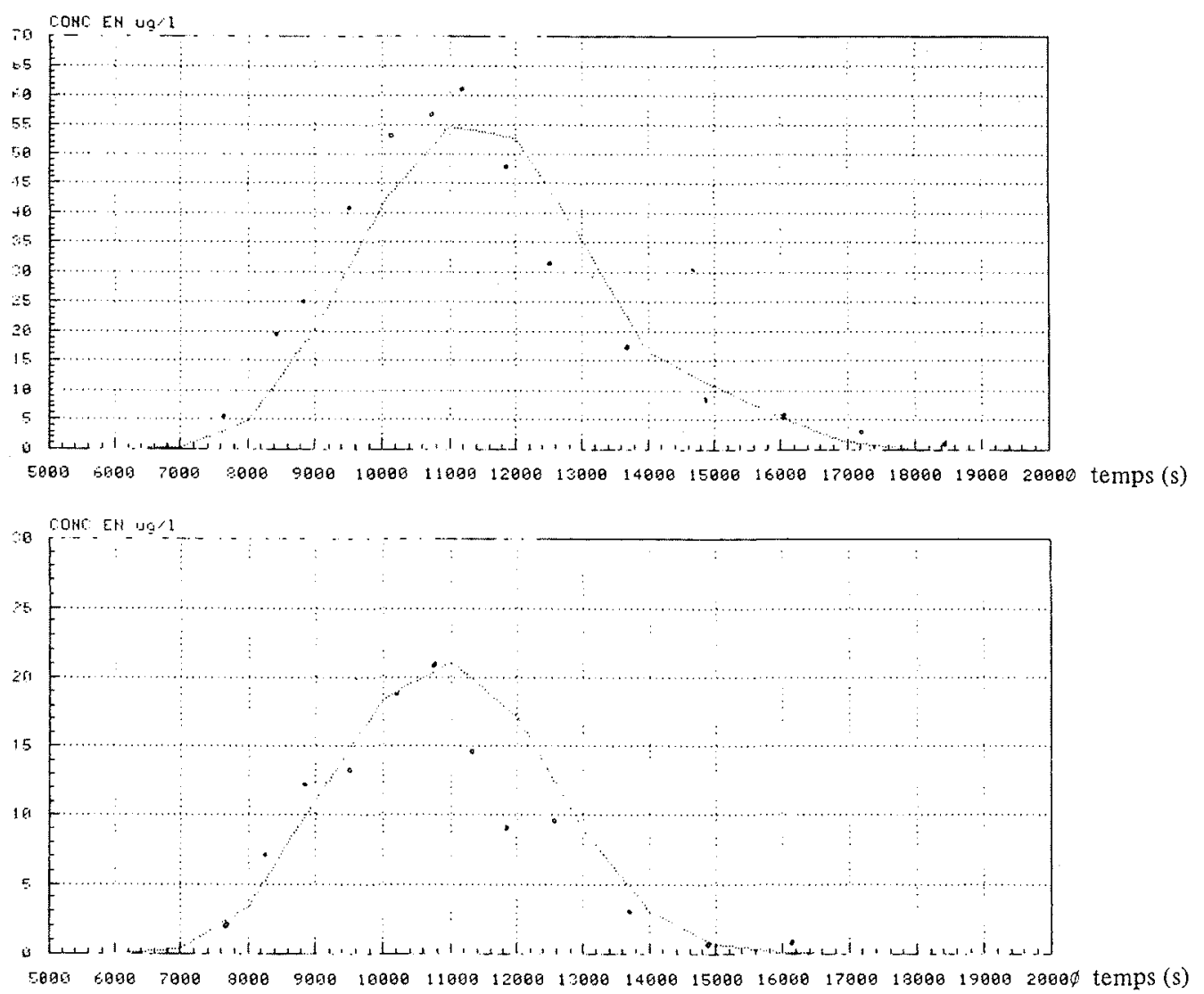

Fig. 5

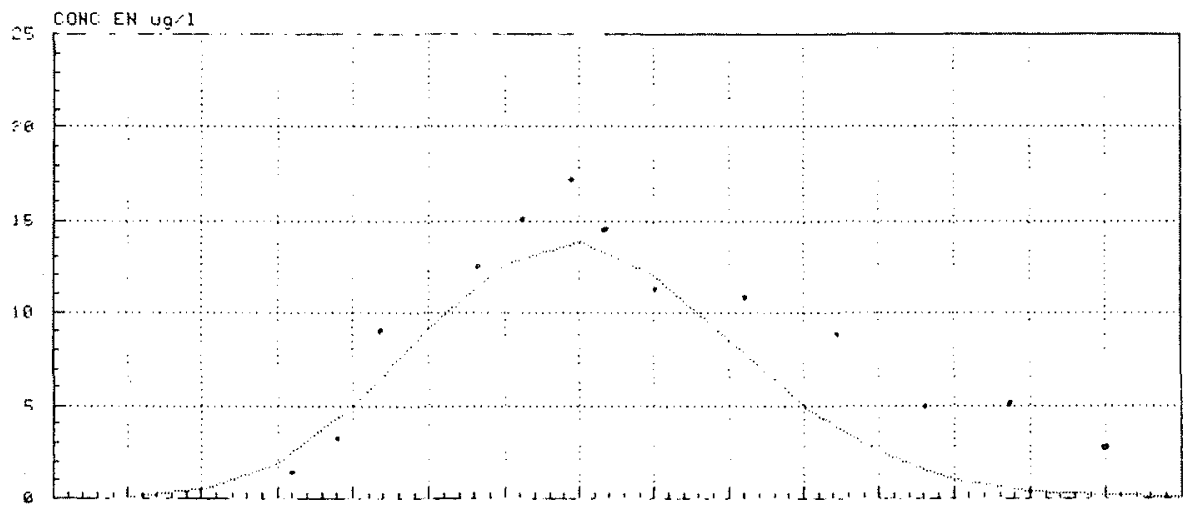

Fig. 4

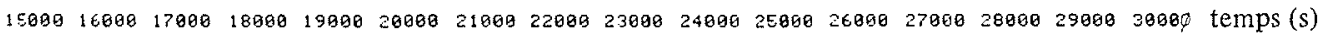

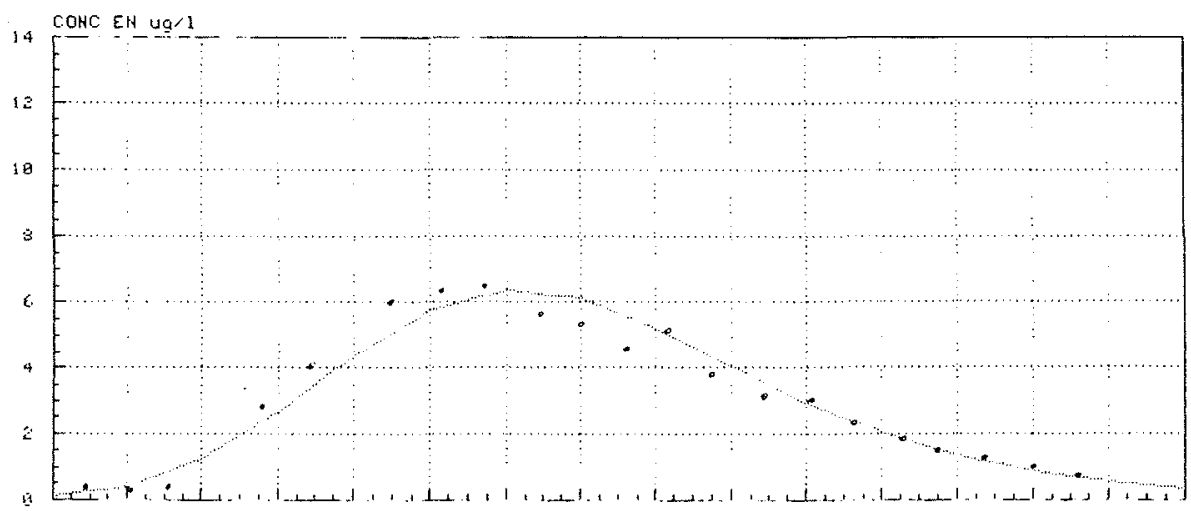

Fig. 6

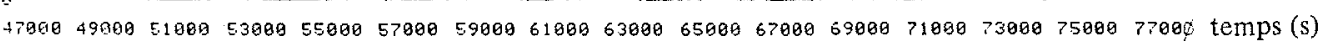

Programme DISPERSO

Simulation Essai du 20/07/1981 - par temps sec - Profils des concentrations calculés aux points de prélèvements suivants:

Figure 4. - Soisy-sur-Seinc. Rive Gauche.

Figure 5. - Soisy-sur-Scine. Milieu.
Figure 6. - Ris-Oranges - Milieu

Figure 7. - Vigneux sur Seine - Rive droite 
sives et par un étalement dans le temps insuffisant. La longue queue de pollution que l'on constate expérimentalement après le passage du maximum de concentration et qui est d'autant plus importante que l'on se place à plus grande distance de la source, n'a pu être rendue de manière fidèle qu'après avoir fait intervenir un certain gradient de la vitesse d'écoulement.

Toutes ces simulations ont été effectuées en limitant la valeur de $R$ à 5 au plus.

\section{Conclusion}

Bien que certains phénomènes d'importance secondaire n'aient pas été pris en compte et malgré l'adoption de quelques hypothèses simplificatrices dans l'établissement du modèle, ce dernier permet de prévoir avec une précision convenable, en cas d'accident de pollution en Seine, l'importance des vagues de pollution pouvant affecter les différentes prises d'eau.

L'emploi du modèle nécessite la connaissance de plusieurs coefficients, dont certains dépendent du débit et des caractéristiques particulières du cours d'eau considéré. Toute application devra donc être précédée d'expériences de traçage à l'aide d'une substance convenable.

Parmi ces coefficients figure également le coefficient de disparition de la substance dont on veut étudier la dispersion et qui est vraisemblablement peu différent de son coefficient de biodégradation (ou qui en dépend fortement); une détermination de ce coefficient, indépendamment du calage du modèle, est donc nécessaire avant tout essai de simulation. (On peut noter à ce sujet qu'une détermination séparée du coefficient de biodégradation de la Rhodamine $B$ aurait simplifié la procédure de calage du modèle exposé plus haut).

Il est tentant d'envisager des améliorations au modèle actuel, l'écueil à éviter sur ce point étant l'élaboration d'un modèle au calage problématique et à l'utilisation délicate. Il n'est pas inutile de souligner que la précision des résultats d'un modèle très élaboré risque d'être illusoire dès lors que son calage est effectué à partir de résultats de mesures inévitablement entâchés d'erreur, notamment lorsqu'il s'agit de résultats de dosages d'autant moins précis que les concentrations sont plus faibles. On peut également s'interroger sur la nécessité d'un modèle très sophistiqué en remarquant qu'en cas de simulation motivée par un accident de pollution, il existera toujours une incertitude sur la quantité de polluant impliquée et sur l'évolution dans le temps du flux parvenant dans le cours d'eau.

\section{Bibliographie}

FISHER H.B. - The mechanics of dispersion in natural streams. Journal of the Hydraulics Division, ASCE, vol 93, $\mathrm{N}^{\circ}$ HY 6 , Proc. Paper 5592, 1967, pp. 187-216.

KRENKEL P.A. - Waste dispersion characteristics of streams using turbulent diffusion phenomenon. J.W.P.C.F., vol 34 $\mathrm{N}^{\circ} 12,1962$, pp. $1203-1212$.

SAYRE W.W. and CHANG F.M. - A laboratory investigation of open channel dispersion processes for dissolved, suspended and floating dispersants. US Géological Survey Professional Paper, 433 E, 1968, 71 pp.

TAYLOR G.I. - The dispersion of matter in turbulent flow through a pipc. Procedings, Royal Society of London, vol 223, Ser A, 1954, pp 446-468. 